\title{
SUPERCLUSTERING AND MOTION OF GALAXY CLUSTERS*
}

\author{
Neta A. Bahcall \\ Space Telescope Science Institute \\ 3700 San Martin Drive \\ Baltimore, MD, USA
}

\begin{abstract}
The evidence for the existence of very large scale structures, $\sim 100 h^{-1} \mathrm{Mpc}$ in size, as derived from the spatial distribution of clusters of galaxies is summarized. A "shell model" of galaxy clustering is described in which clusters of galaxies are located at shell intersections; the model yields results consistent with cluster observations. Detection of $a \sim 2000 \mathrm{~km} \mathrm{~s}^{-1}$ elongation in the redshift direction in the distribution of the clusters is also described. Possible causes of the effect are peculiar velocities of clusters on scales of $10-100 h^{-1} \mathrm{Mpc}$ and geometrical elongation of superclusters. If the effect is entirely due to the peculiar velocities of clusters, then superclusters have masses of order ${ }^{10^{16.5}} M_{\odot}$ and may contain a larger amount of dark matter than previously anticipated.
\end{abstract}

\section{INTRODUCTION}

Rich clusters of galaxies are an efficient tracer of the large scale structure in the universe. Very large structures, of size $\sim 100 h^{-1} \mathrm{Mpc}$ or more, show up in the distribution of the rich clusters. ( $h \equiv H_{0} / 100 \mathrm{~km} \mathrm{~s}^{-1} \mathrm{Mpc}^{-1}$ is used throughout this paper.) Clusters have mean separations of order $50 \mathrm{~h}^{-1} \mathrm{Mpc}$ and are therefore efficient in revealing these large scale structures that were not detected previously in the distribution of individual galaxies. The existence of large scale structures became evident by observing strong correlations among clusters, correlations that extend to separations as large as $100 \mathrm{~h}^{-1}$ Mpc (Bahcall and Soneira 1983; Klypin and Kopylov 1983; Hauser and Peebles 1973), as well as by determining a specific catalog of superclusters in three dimensions (Bahcall and Soneira 1984). Other evidence, such as a giant void $\left(\sim 300 h^{-1} \mathrm{Mpc}\right)$ of rich clusters (Bahcall and Soneira 1982b), and the extension of some rich superclusters to at least $100 h^{-1} \mathrm{Mpc}$ scale (Bahcall and Soneira 1982a), further support the existence of very large scale structures as a common feature in the universe. Work in progress is 
described below in which we show that a "shell model" of galaxy clustering can describe the known cluster observations. In this model, the clusters of galaxies are located at shell intersections.

The structure work was recently extended by Bahcall, Soneira, and Burgett (1986), who used the rich clusters to determine whether peculiar velocities exist on large scales. We find evidence for a large velocity broadening in the redshift distribution that corresponds to a cluster velocity of $\sim 10^{3} \mathrm{~km} \mathrm{~s}^{-1}$. This suggests that dark matter may dominate even on these very large scales.

In this review I summarize the superclustering and motion of clusters of galaxies

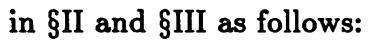

Section II: Superclustering of Galaxy Clusters

A. Superclusters and Voids

B. The Cluster Correlation Function

C. Phenomenological Clustering Models: Tails and Shells in the Galaxy Distribution

Section III: Motion of Clusters

A. Peculiar Velocities Among Clusters

B. Geometrical Elongation of Superclusters

\section{SUPERCLUSTERING OF GALAXY CLUSTERS}

\section{A. Superclusters and Voids}

A complete catalog of superclusters (Bahcall and Soneira 1984), defined as groups of rich clusters of galaxies and identified by a spatial density enhancement of clusters, reveal significant structures, over random, to scales of $\sim 100 h^{-1} \mathrm{Mpc}$. The supercluster catalog was used by Bahcall and Soneira (1982a) to study the area around the large void of galaxies in Bootes observed by Kirshner et al. (1981). It was found that the largest, densest superclusters are located near and around the void. In addition, the overdensity of galaxies observed by Kirshner et al. on both redshift sides of the $z \simeq 0.04-0.06$ void was found to coincide in redshift space with the nearby dense superclusters. This suggests that the large superclusters surround the giant galaxy void and that the tails of their

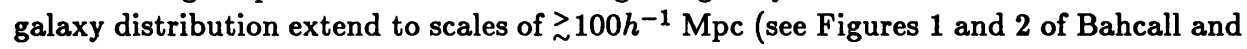
Soneira 1982a).

Another indication of a very large scale structure, or density fluctuation, is the huge void of cataloged nearby rich clusters of galaxies observed in the complete $D \leq 4$ Abell (1958) sample (Bahcall and Soneira 1982b). The void is in the approximate redshift range of $z \simeq 0.03-0.08$, and it extends $\sim 100^{\circ}$ across the sky (i.e., $\sim 300 h^{-1} \mathrm{Mpc}$ ). Its projected area is completely devoid of nearby - but not distant - rich clusters $(R \geq 1)$. If this apparent void in nearby rich clusters is real, it subtends a volume of more than $10^{6} h^{-3} \mathrm{Mpc}^{3}$. 


\section{B. The Cluster Correlation Function}

\section{Clusters of Galaxies}

The spatial correlation function of rich clusters of galaxies (Bahcall and Soneira 1983) reveals strong correlations among clusters and implies the existence of structures to scales of at least $\sim 100 h^{-1} \mathrm{Mpc}$. Consistent results have been obtained by other investigators (Hauser and Peebles 1973; Klypin and Kopylov 1983; Shvartsman, this proceedings). The above investigation is based on studying all rich clusters in the complete redshift sample of the Abell (1958) catalog to distance group $D \leq 4$ (i.e., $z_{\sim}^{<0.08) . ~ T h e ~ s t u d y ~}$ also includes, as a test, the determination of the angular correlation function of all 1651 clusters in the entire Abell catalog to $D \leq 6\left(z_{\sim}^{<0.2)}\right.$.

The cluster correlation function is observed to be approximately 18 times stronger than the correlation function of galaxies (Figure 1). The cluster correlations, $\xi_{c c}$, for Abell richness groups $R \geq 1$ clusters can be expressed as (Bahcall and Soneira 1983):

$$
\xi_{c c}(r)=360 r^{-1.8} \quad r_{\sim}^{<100 h^{-1} \mathrm{Mpc}}
$$

while the galaxy correlation function (e.g., Groth and Peebles 1977) is represented by:

$$
\xi_{g g}(r)=20 r^{-1.8} \quad r_{\sim}^{<20 h^{-1} \mathrm{Mpc}}
$$

Bahcall and Soneira (1983) also found that the correlation function depends strongly on cluster richness, with rich clusters $(R \geq 2)$ showing stronger correlations by a factor of $\sim 3$ as compared with the poorer $(R=1)$ clusters (both are consistent with an $r^{-1.8}$ power law). This result, combined with the lower correlation amplitude of individual galaxies lead the authors to the conclusion that progressively stronger correlations exist, at a given separation, for richer galaxy systems. This trend of increasing correlation strength with richness is shown in Figure 2. A recent study by Shectman (1985) of the correlations of still poorer clusters appears to be consistent with the trend suggested by Bahcall and Soneira. A phenomenological model that can explain the observed increase of correlation strength with richness was recently proposed by Bahcall (1986), and expanded by Bahcall, Henriksen, and Smith (1987); both models are summarized in $\S \mathrm{IIC}$.

A representation of the observed correlations as a universal dimensionless correlation function, and its implication for a scale invariant clustering process is discussed in §IIB3.

\section{Superclusters}

Bahcall and Burgett (1986) carried the study of rich galaxy clusters one step further by studying the spatial distribution of superclusters. The sample used was the BahcallSoneira (1984) complete catalog of superclusters to $z \leq 0.08$.

The results reveal correlations among superclusters on a very large scale: $\sim 100$ $150 h^{-1} \mathrm{Mpc}$. Because of the small size of the supercluster sample, the statistical uncertainty is appreciable; the observed effect is at the $3 \sigma$ level. The supercluster correlation 
strength is stronger than that of the rich cluster correlations by a factor of approximately 4. It is approximately two orders of magnitude stronger than the galaxy correlation amplitude. While this enhancement is observed in the $\sim 100-150 h^{-1} \mathrm{Mpc}$ range, it is possible that the supercluster correlation function also follows an $r^{-1.8}$ law. The increase in correlation strength for superclusters is consistent with the predicted increase of correlation strength with richness (luminosity) of the system (Figure 2).

\section{A Universal Correlation Function}

The increase of correlation strength with richness (Figure 2) implies that rich, luminous systems are more strongly clustered, at a given separation, than poorer systems. The power-law of the correlation functions is also observed to be identical in the various systems studied. Either initial conditions, or superceding evolution, may be responsible for these observed clustering phenomena.

In Figure 3 we plot the amplitude of the correlation functions of the various systems (galaxies, poor and rich clusters, superclusters) as a function of the mean separation of objects in the sample, $d$ (see Bahcall and Burgett 1986). The mean separation is related to the mean spatial density of objects in the sample, $n$, through $d=n^{-\frac{1}{3}}$. It is apparent from Figure 3 that the correlation strength increases with the sample's mean separation. Moreover, a dimensionless correlation function normalized to the sample's mean separation, $d$, appears to yield a constant, universal function for all systems studied (with some enhancement for galaxies, as described below). This universal dimensionless correlation function has the form

$$
\xi_{i}(r) \simeq 0.3\left(r / d_{i}\right)^{-1.8},
$$

where the index $i$ refers to the system being considered, and $d_{i}$ is its mean separation. The correlation function of galaxies is stronger than given by equation (3) by a factor of about four (see also Figure 3).

The universality of the correlation function implies a scale-invariant clustering process (Szalay and Schramm 1985). The stronger dimensionless galaxy correlations may imply gravitational enhancement on smaller scales. If a non-linear process, other than gravity, participates in galaxy formation, and this process is scale-invariant, the created structure will have a single power-law correlation function, the slope of which is related to the geometry of the structure, i.e., its fractal dimension. Small scale gravitational clustering may break the scale invariance and increase the dimensionless correlation amplitude for galaxies.

Cosmic strings, serving as the primary agent in the formation of galaxies and clusters, was suggested as one model for such a scale-invariant infrastructure (Turok 1986). The model yields a scale-invariant correlation function similar to that observed, with a power-law of $\mathbf{- 2}$.

\section{Supporting Evidence for the Cluster Correlation Function}

Several checks support the findings discussed above for the cluster correlation function; these checks are summarized below. 
- The angular cluster correlation function scales with depth as expected from real spatial correlations, rather than from selection on biases (Bahcall and Soneira 1983; Hauser and Peebles 1973).

- The cluster correlation function yields consistent results in different large regions of the sky (e.g., north versus south, high versus low latitudes, different longitude ranges; Bahcall and Soneira 1983).

- The projected and redshift cluster correlation functions yield consistent results, thus strongly supporting the reality of the correlations.

- The cluster correlation as determined from the Abell sample is consistent with more recent results using other samples and catalogs (e.g., Shectman 1985; Zwicky clusters - Postman et al. 1986; and sub-samples of different regions and redshifts in the Abell catalog - Shvartsman, this proceedings).

- The cluster correlation function is consistent with the galaxy-cluster cross-correlation function determined by Seldner and Peebles (1977). The latter is less sensitive to the Abell catalog than the former.

- A preliminary estimate of the completeness limit of the nearby Abell sample obtained by comparisons with X-ray data of clusters yields a reasonably high completeness level (work in progress in collaboration with Gioia et al.).

\section{Phenomenological Clustering Models: Tails and Shells in the Galaxy Distribution}

\section{Long Tails to Galaxy Clusters}

A phenomenological model that explains the observed difference between the galaxy and rich cluster correlation functions was recently proposed by Bahcall (1986). The model is based on the fraction of galaxies that participate in the clustering, and explains the observed trend of increased correlation strength with richness. A summary of the model is presented below.

The galaxy correlations depend, at least partially, on the rich cluster correlations since clusters contain galaxies. It can be shown that if all galaxies were members of rich clusters, the two correlation functions should be approximately the same on large scales. In this case they both trace the same large scale structure. The fraction of galaxies in clusters is clearly less than unity. The effect of the fraction of galaxies, $f$, that are associated with rich clusters, on the relation between the cluster and galaxy correlation functions, is the basis for the model. The parameter $f$ represents the probability that a randomly chosen galaxy is correlated with a rich cluster. These associations may include large structures (separations of tens of $\mathrm{Mpc}$ ), comparable to the separations observed in the cluster correlation function (and well above the standard Abell radius of $1.5 h^{-1}$ Mpc).

The galaxy correlation function contains contributions from three terms: galaxy pairs from the fraction $f$ of galaxies that are cluster members; pairs from the fraction 1- $f$ of galaxies that are non-cluster members ("field"); and cross-term pairs. Inserting 
the analytic expressions for each of these terms into the expression for the overall galaxy correlation function yields:

$$
\left(\frac{\xi_{c c}}{\xi_{g g}}\right)^{\frac{1}{2}}=\frac{1-(1-f)\left(\xi_{g g}^{f} / \xi_{g g}\right)^{\frac{1}{2}}}{f}
$$

The above ratio of the cluster to galaxy correlation strength depends on two parameters: the fraction of galaxies in clusters, $f$, and the ratio of the "field" galaxy correlation strength, $\xi_{g g}^{f}$ (i.e., the correlation of the 1- $f$ fraction of galaxies outside the rich clusters) to the overall galaxy correlation $\xi_{g g}$. If all galaxies were associated with rich clusters, i.e., $f=1$, then the galaxy and cluster correlations are identical, as expected. However, for any fraction $f<1$, the galaxy correlations will be smaller than the parent cluster correlations due to the reducing effect of the less clustered "field" galaxies. It is found that if approximately $20 \%$ of all galaxies are associated with rich $(R \geq 1)$ clusters, the galaxy correlation function will be, as observed, $\sim 18$ times weaker than the cluster correlations.

The model suggests that the fraction of galaxies associated with rich clusters is considerably larger than previously expected; most of these galaxies are distributed in the outer tails of the clusters, which may extend to at least $\sim 30 h^{-1} \mathrm{Mpc}$. Most clusters are therefore predicted to be embedded within much larger structures.

\section{The Shell Model}

An extension of the phenomenological model discussed above is a specific case in which galaxies are distributed on surfaces of shells (or cells), with rich clusters located at shell intersections. Such a picture may be suggested by redshift surveys of galaxies (Gregory et al. 1981; de-Lapparent et al. 1986; Giovanelli et al. 1986). In order to test this model and its agreement with the observed galaxy and cluster correlations, we (Bahcall, Henriksen, and Smith 1987) placed galaxies on surfaces of randomly placed shells, and formed clusters at the shell intersections. The range of parameters for shell sizes and number density was varied. The galaxy and cluster correlation functions were determined and compared with observations. We find that the model cluster correlations are consistent with the observed cluster correlations, showing the large increase in correlation strength from galaxies to clusters (§IIB). This main result appears to be basic to the model and is not very sensitive to the exact parameters used. The model galaxy correlations appear to be consistent with observations on small scales, but exhibit a tail of weak positive correlations at larger separations not seen in the data.

An example of a typical model result is shown in Figure 4. More detailed results are given in Bahcall et al. (1987). The results therefore suggested that the strong observed cluster correlations may be simply due to the geometry of clusters positioned on randomly placed shells or similar structures; the typical structure size is best fit with a radius of approximately $20 h^{-1} \mathrm{Mpc}$. 


\section{MOTION OF CLUSTERS}

The discussion in the previous sections summarizes evidence for the general existence of structures on the scale of $\sim 10-150 h^{-1} \mathrm{Mpc}$. A question of critical importance is what are the velocity fields in these structures. Peculiar velocities of clusters on these scales may indicate the existence of large amounts of (dark) matter. Recently, Bahcall et.al. (1986) used the complete redshift sample of rich clusters (§II) to study the possible existence of peculiar motion and/or structural anistropy on large scales. We find strong broadening in the redshift distribution that corresponds to a cluster velocity of $\sim 10^{3}$ $\mathrm{km} \mathrm{s}^{-1}$. These findings are summarized below.

The distribution of clusters in space was studied by separating the three-dimensional distribution into its components along the line-of-sight (redshift) axis and the perpendicular axes projected on the sky $(\alpha$ and $\delta$ ). All clusters were assumed to be located at their Hubble distances as indicated by their redshifts, and their pair separations in Mpc were determined in the three components. A scatter-diagram of the cluster pair separations in the $z$ direction $\left(R_{z}\right)$ versus their separations in $\alpha$ or $\delta\left(R_{\alpha}\right.$ or $\left.R_{\delta}\right)$ was then determined for each sample.

If all clusters were located at their Hubble distances with negligible peculiar motion, and if the sample was not dominated by elongated structures in a given direction, a symmetric scatter-diagram should be observed. If a large peculiar velocity exists among clusters, it would manifest itself as an elongated distribution along the $z$-direction in the $R_{z}-R_{\alpha}$ and $R_{z}-R_{\delta}$ diagrams. Such an elongation is normally interpreted as peculiar motion. However, the effect may also be caused by geometrically elongated structures, if they dominate the sample (with elongation toward the $z$-direction; see below).

The results are presented in Figure 5. A strong and systematic elongation in the $z$-direction exists in all the real samples studied. Scatter-diagrams for sets of random catalogs do not exhibit any conspicuous elongation. The effect of elongation is strong; statistically it corresponds to approximately $8 \sigma$ in a single sample (assuming, for illustrative simplicity, Gaussian statistics). It is therefore unlikely that the observed redshift elongation is a chance fluctuation. The effect becomes more apparent in the larger $R \geq 0$ sample; this is expected if the effect is real.

Convolving the frequency distribution along the projected axis with a Gaussian velocity distribution to match the broadened redshift distribution, yields a velocity width of $\sqrt{2} \sigma \simeq 2000 \mathrm{~km} \mathrm{~s}^{-1}$ for cluster pairs.

The observed elongation may be caused by either peculiar motion of clusters or a true geometrical elongation of superclusters. These are briefly discussed below.

\section{A. Peculiar Velocity Among Clusters}

To estimate a supercluster mass which may support this velocity, we use a typical supercluster size of $\sim 25 h^{-1} \mathrm{Mpc}$ (=cluster correlation scale-length) and assume the virial relation $M \propto v^{2} r$. This yields a typical supercluster mass of

$$
M_{s c} \simeq 3 \times 10^{16} M_{\odot}
$$


This mass is comparable to the mass of $\sim 30$ rich clusters while typically only $\sim$ 3-5 rich clusters are members of a supercluster. Even when the luminous tails of clusters are accounted for the result may still imply an excess of dark matter in superclusters as compared with clusters. Using an observed luminosity and/or density profile of $r^{-3}$ or $r^{-2.5}$ around a rich cluster, we estimate an $M / L$ for superclusters that is typically two to three times that of rich clusters, i.e., $M / L \sim 500$.

\section{B. Geometrical Elongation of Superclusters}

The elongation observed in the scatter diagrams may also be caused, at least partially, by a geometrical elongation of superclusters. If the most prominent superclusters are elongated toward the line-of-sight direction, an apparent elongation in the distribution of pair separation along this axis may result. Accurate distance indicators such as TullyFisher type relations are needed in order to distinguish between peculiar velocity and geometrical elongation of large scale structures.

\section{$\underline{\text { References }}$}

Abell, G. O., 1958, Ap. J. Suppl. 3, 211

Bahcall, N. A., 1986, Ap. J. Letters 302, L41.

Bahcall, N. A., and Burgett, W. S., 1986, Ap. J. Letters 300, L35.

Bahcall, N. A., and Soneira, R. M., 1982a, Ap. J. Letters 258, L17.

Bahcall, N. A., and Soneira, R. M., 1982b, Ap. J. 262, 419.

Bahcall, N. A., and Soneira, R. M., 1983, Ap. J. 270, 20.

Bahcall, N. A., and Soneira, R. M., 1984, Ap. J. 277, 27.

Bahcall, N. A., and Soneira, R. M., and Burgett, W. S., 1986, Ap. J. $311,15$.

Bahcall, N. A., Henriksen, M. J., and Smith, T. E., 1987, to be submitted to Ap. J. de-Lapparent, V., Geller, M., and Huchra, J., 1986, Ap. J. Letters 302, L1.

Giovanelli, R., Haynes, M., and Chincarini, G., 1986, Ap. J. 300, 77.

Gregory, S. A., Thompson, L. A., and Tifft, W. G., 1981, Ap. J. 243, 411.

Groth, E., and Peebles, P. J. E., 1977, Ap. J. 217, 385.

Hauser, M. G., and Peebles, P. J. E., 1973, Ap. J. $185,757$.

Kirshner, R. P., Oemler, A. Jr., Schechter, P. L., and Shectman, S. A., 1981, Ap. J. Letters 248, L57.

Klypin, A. A., and Kopylov, A. I., 1983, Soviet Astronomy Letters 9, 41.

Postman, M., Geller, M., and Huchra, J., 1986, A.J. 91, 1267.

Shectman, S., 1985, Ap. J. Suppl. 57, 77.

Seldner, M., and Peebles, P. J. E., 1977, Ap. J. 215, 703.

Szalay, A. S., and Schramm, D. N., 1985, Nature 314, 718.

Turok, N. 1986, preprint. 

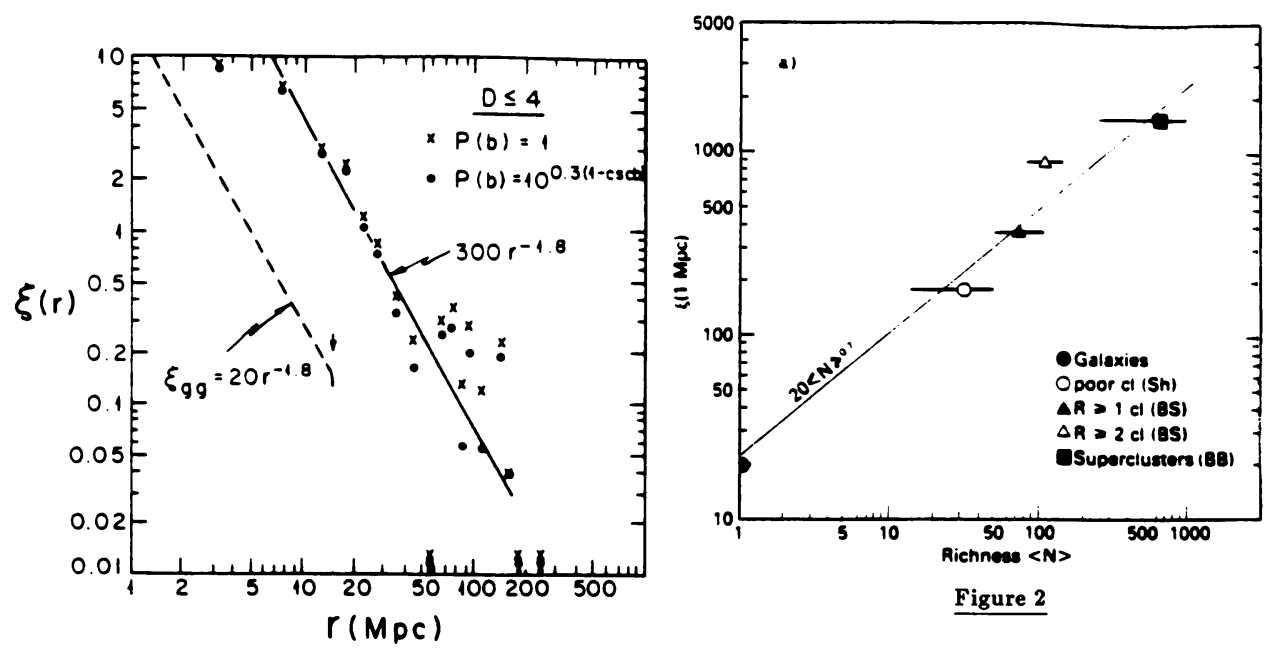

Figure 1:

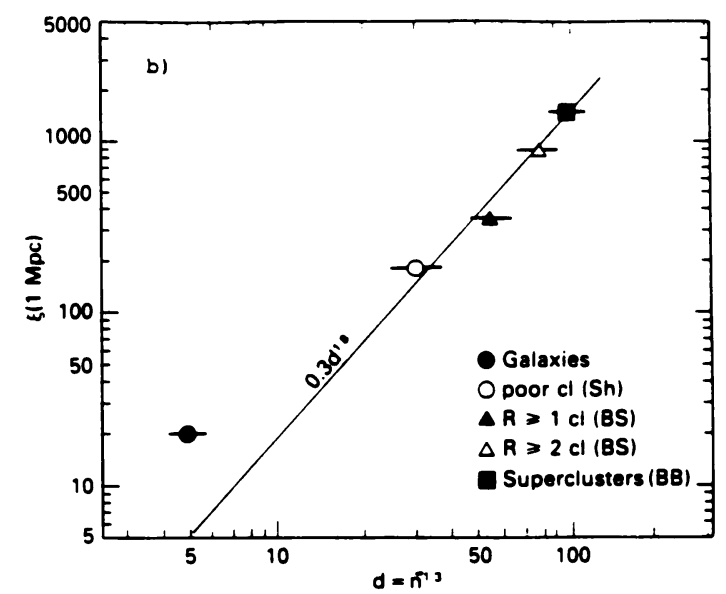

Figure 3:

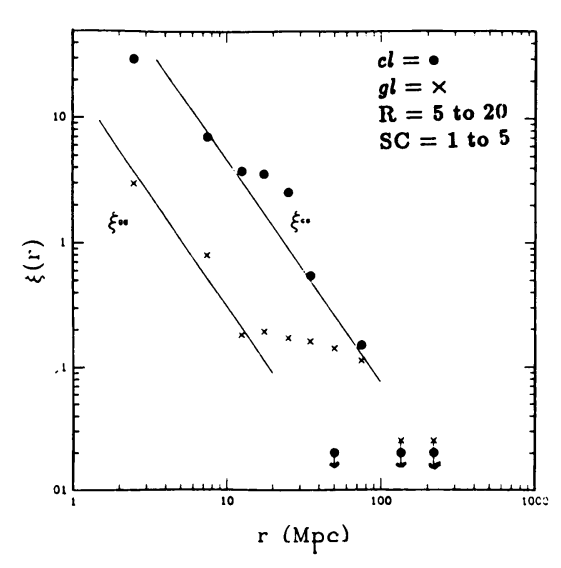

Figure 4:

Figure 1: The spatial correlation function of the $D \leq 4$ Abell sample (Bahcall and Soneira 1983). The dashed line is the galaxy-galaxy correlation function determined by Peebles and co-workers.

Figure 2: The dependence of the correlation function on the mean richness ( $\propto$ luminosity) of the system (Bahcall and Burgett 1986). the points are for galaxies, poor clusters (Schectman 1985), $R \geq 1$ and $R \geq 2$ Abell clusters (Bahcall and Soneira 1983), and superclusters (Bahcall and Burgett 1986). The solid line indicates the approximate dependence of richness.

Figure 3: The dependence of the correlation function on the mean separation of objects in the system (Bahcall and Burgett 1986). The solid line represents a $d^{1.8}$ dependence.

Figure 4: Shell-Model correlation functions for clusters (dots) and galaxies (crosses), and their comparisons with observations ( $\xi_{g g}$ and $\xi_{c c}$ lines). The plotted model represents shell radii distribution in the range 5 to $20 \mathrm{~h}^{-1}$ $\mathrm{Mpc}$, and,depending on size, 1 to 5 clusters forming at shell intersection (Bahcall et al. 1987). 

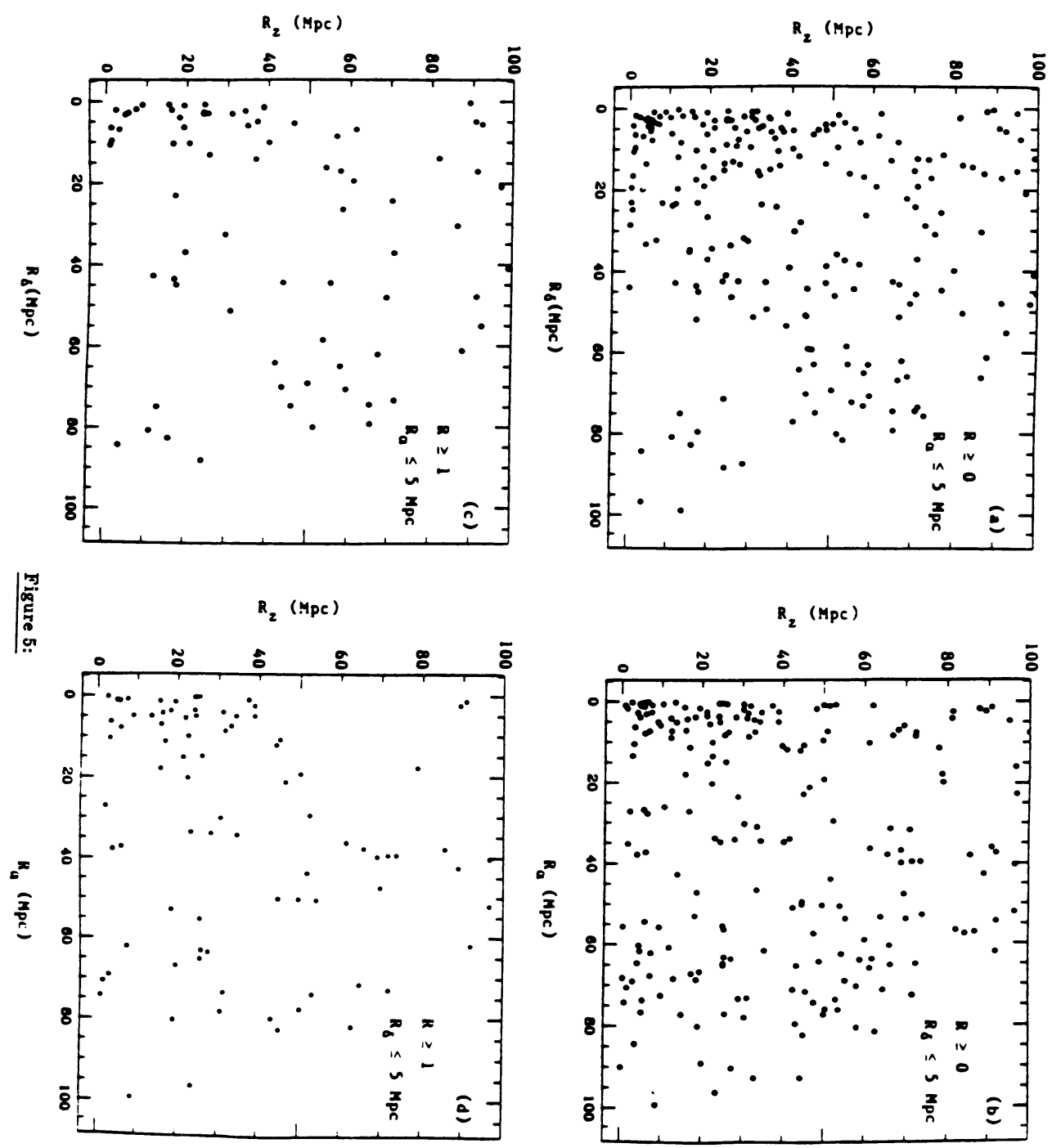

Figure 5: Scatter-diagrams of cluster pair separations in $M p c$ in the $R_{z}-R \alpha$ and $R_{z}-R \delta$ planes (Bahcall et al. 1986). Figure 6a-6b and $6 c-6 d$ represent, respectively, the $R \geq 0$ and $R \geq 1$ richness samples. The elongation in the redshift direction is apparent in all cases. 